\title{
Salvaging God's Own Creation: Reattach the Broken-A Case Report
}

\author{
Nimisha $\mathbf{S}^{1 *}$, Vidhi $\mathbf{S}^{2}$ and Meghna $\mathbf{K}^{3 *}$ \\ ${ }^{1}$ Professor and Head of the Department, KM Shah Dental College and Hospital, Sumandeep \\ Vidyapeeth, India \\ ${ }^{2}$ Post graduate student (MDS), KM Shah Dental College and Hospital, Sumandeep Vidyapeeth, \\ India \\ ${ }^{3}$ Post graduate student (MDS), KM Shah Dental College and Hospital, Sumandeep Vidyapeeth, \\ India
}

\section{Case Report}

Volume 5 Issue 2

Received Date: April 20, 2020

Published Date: May 11, 2020

DOI: $10.23880 /$ oajds-16000254

*Corresponding author: Nimisha Shah, Professor and Head of the Department, KM Shah Dental College and Hospital, Sumandeep Vidyapeeth, Vadodara, Gujarat, India, Tel: +91 9998994299; Email: nshah7873@gmail.com

\section{Abstract}

Fracture of anterior teeth is most common type of dento-alveolar traumatic injuries. It mostly affects upper anterior teeth due to its exposed position in the dental arch which further leads to psychological stress and anxiety to patient. There have been various treatment modalities for these type of complicated crown root fractures. This case report describes multidisciplinary approach for fragment re attachment of maxillary permanent central incisior using dentin adhesives. A 25 year old, patient reported with oblique fracture of upper right central incisior with fracture line extending subginvially in palatal aspect. The margins were re-established using full thickness mucoperiosteal flap. The broken fragment was re attached with a glass fibre post to increase the retention. On further follow up, it establishes an esthetic and successful outcome of this conservative approach.

Keywords: Traumatic injuries; Fragment re-attachment; Glass fibre post; Ultra conservative approach

\section{Introduction}

Traumatic injuries are the most common rationale for crown fractures in the permanent dentition. A comprehensive treatment plan is an essential prerequisite towards successful rehabilitation of complicated fractures of anterior teeth. While it is imperative to restore the biological, functional and esthetic form, adequate consideration must also be given to the patient's desire and his or her attitude towards treatment [1]. Coronal fractures are most common type of dental injuries. Investigation into incidence of trauma has made it clear that complicated crown root fracture is significant in number. The common reasons attributed to this are falls, occupational injuries, high-impact sports, and motor vehicle accidents. Most commonly affected tooth is the maxillary central incisor, followed by lateral incisor which sustains approximately $92 \%$ of the dental injuries [2]. Maxillary incisors are more susceptible to get damaged by a blow on the face due to their labial projection in arch.

Fractures subsequently lead to aesthetic, functional, and phonetic problems. Choosing the correct treatment to be followed is based on the age of the dental patient; the extent of the fracture (severity and location of the invasion of the biologic width); the presence or absence of endodontic involvement; the presence/absence of the tooth fragment and its condition of use; the occlusion and esthetics; and time and patient expectations [3]. With the improvement of adhesive materials, reattachment of tooth fragment has become a more predictable technique with several advantages. It is a conservative procedure that maintains the original tooth contour, texture and translucence; is economical and obtains 
esthetics in a single appointment. Subgingival tooth fractures can also be restored successfully with this method [4]. The present case report describes reattachment procedure successfully carried out in anterior teeth with oblique fractures occurring at the subgingival level.

\section{Case Report}

A 25-year-oldmale presented to the Department of Conservative Dentistry and Endodontics with a chief complaint of fracture of upper front tooth caused by an occupational injury 1 day prior. The patient had no significant past dental history. Further history suggested of no signs of bleeding, sinus tract or any soft tissue laceration. Clinical examination revealed fragment was in place which could be separated with help of probe. Pulpal involvement was seen following separation of fractured fragment, and the fracture line was sub gingival. Radiographic examination revealed a complicated oblique crown fracture that extended subgingivally on the palatal area of the tooth.

Periodontal condition was healthy enough to allow reasonable longetivity of the restoration. Remaining crown structure was healthy and one third of the length of the tooth. Fracture line was extending sub gingival but was supracrestal and patient's main interest was in retaining its own natural tooth. Reattachment of the fragment along with glass fiber and fiber reinforced composite was the optimal mode of treatment for the present case (Figure 1).

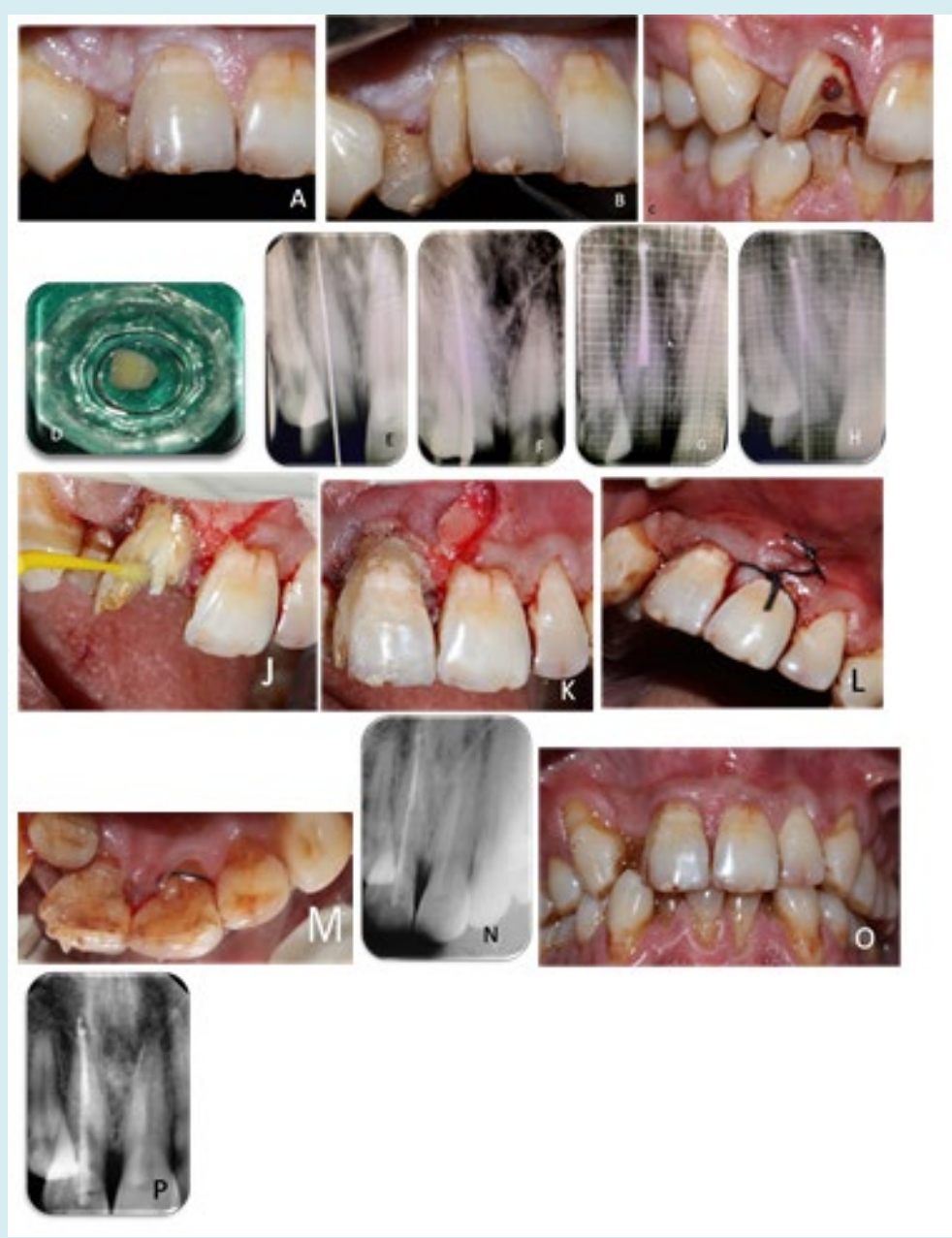

Figure 1: A) Pre-operative clinical picture, B) Separation of fracture fragment with straight probe, C) After removal of fracture fragment, D) Fragment stored in saline, E) Radiographic confirmation of working length, F) Master cone verified on radiograph, G) Obturation, H) Post space preparation and selection of fibre post evaluated on radiograph, I) Full thickness mucoperiosteal flap raised, J) Cementation of glass fibre post and application of bonding agent , K) Cementation of fragment with fibre reinforced composite, L) Single interrupted sutures placed, M) Immediate postoperative view, N) post-operative radiograph, 0-P) 6 follow up. 


\section{Open Access Journal of Dental Sciences}

During its removal, it was observed that the fracture line was extending subginigival in palatal area. After the tooth fragment was removed under local anesthesia (Nirlife) $1.8 \mathrm{ml}$ lidocaine 1:200000was injected in bilateral infra orbital nerve block and $0.8 \mathrm{ml}$ for nasopalatine nerve block. It was stored in normal saline solution $(0.9 \% \mathrm{w} / \mathrm{v}$, Otsuka pharmaceutical India Pvt Ltd) till completion of the treatment. The clinician opted to perform a single-session endodontic treatmentirtwith11.Working length evaluated on an electronic apex locator (J Morita) and on radiograph, was $16.5 \mathrm{~mm}$ and biomechanical preparation done with hand $\mathrm{K}$ files (Mani) rotary files (neo-endo). Following sectional obturation till $10 \mathrm{~mm}$ length was done, with remaining post space available was $6-7 \mathrm{~mm}$. Post space preparation was done with peso reamer of size no.3(Mani). Subsequently, prefabricated glass fiber post of size 1.1(Angelus) was selected and confirmed on radiograph. Further, the canal was irrigated with EDTA liquid (PREVEST Dent Pro), followed by normal saline solution. Since the fracture line was subgingival full thickness mucoperiosteal flap was raised to expose the margins of the tooth. Cervicular incision was made extending from distal of 11 to mesial of 21with Bard Parker handle and surgical blade no 15. Flap elevated with periosteal elevator. Complete margins of the tooth were visible and hemostasis was achieved with gauze soaked in adrenaline. Before, cementation, the canal was dried with paper points (Dia dent). Fibre point was cemented in the canal with resin based adhesive cement (Calibra, Dentsply).

Dimples were prepared in the fractured fragment and the tooth as well with carbide round bur no 2 before cementation of post for additional retention and were made on the opposite side of both the structures. The fragment was etched with $37 \%$ phosphoric acid ( $d$ tech) beyond the margins for 15 seconds and rinsed with air/water spray for 15 secs. After being dried, two layers of the bonding agent (Solare universal Bond, GC) were applied and thinned with air jets. The fragment was preserved, without light activation, protected from the ambient light. On the other hand, the tooth was also etched with $37 \%$ phosphoric acid for 15 seconds. After being rinsed for 30 seconds, the enamel surface was left completely dry, while dentin was left slightly moist. Two layers of an adhesive were applied. Subsequently, resin cement (Calibra, Dentsply) was used to fill the hole in the tooth and the prepared grooves into the coronal fragment. Additional reinforcement was done by placing fiber reinforce flowable composite (GC, Europe) along with resin cement. The fragment was carefully seated on the remaining tooth and light cured for 20 seconds $(900 \mathrm{~mW} /$ $\mathrm{cm} 2$ output). During curing, firm and stable finger pressure was applied to the coronal fragment to closely adapt it to the tooth. Full thickness mucoperiosteal flap was closed with single interrupted sutures. The excess cement was removed and the tooth was reduced and was made out of occlusion.
Immediate post-operative IOPA was taken to check for the adaptation. Patient was recalled after 7 days for clinical evaluation and suture removal. Patient was recalled for follow up at an interval of 3 months and 6 months.

\section{Discussion}

Traumatic injuries involving tooth fracture can now be treated by reattachment of the tissue fragments using an adhesive system to provide what is considered to be the ultra-conservative mode of restorations [5]. The development of adhesive material creates new perspective in the reconstruction of fractured teeth; it is now possible to achieve excellent results with the reattachment of dislocated tooth fragment provided that the biological factors, materials and techniques are logically assessed and managed. Survival rates for such restorations have been shown to be good, with failure often only resulting from subsequent trauma.

Factors which influence the management of coronal tooth fractures are: [6]

a) Extent of fracture (biological width, endodontic involvement, alveolar bone fracture).

b) Pattern of fracture and restorability of fractured tooth (associated root fracture).

c) Secondary trauma injuries (soft tissue status).

d) Presence/absence of fractured tooth fragment and its condition for use (fit between fragment and the remaining tooth structure).

e) Occlusion, aesthetics Prognosis and extra oral time

f) Reattachment of fractured tooth fragments offers a viable restorative option for the clinician because it restores tooth function and esthetics with the use of a very conservative and cost-effective approach.

Reattachment is a mode of preferred treatment because of wear rate similar to adjacent/opposed teeth, colour match to the remaining crown portion, preservation of incisal translucency/good aesthetics, maintenance of natural tooth contours, more durable restoration than a class IV resin restoration alone, preservation of 'identical' occlusal contacts, colour stability of the enamel, positive emotional and social response from patients [7]. The successful reattachment depends on the extent of dehydration of the fragment and reinforcement techniques. Dehydration of the fragment leads to altered esthetics and results in poor bond strength, hence storage of the fragment was done in saline solution. In this case, we have used an adhesive, a dual-curing luting composite system, a glass-fiber-reinforced composite root canal post, fibre reinforced composite and the original crown fragment were used. This technique provides reinforcement to the restored segments and increases durability. Glass fiber post was used in present case as it creates aninter lock mechanism between the two fragments and minimizes the stresses on the reattached tooth fragment. As, modulus of 
elasticity is same as that of dentin so requires minimal or no preparation and creates a mono-block effect [8-10].

Fiber reinforced flowable composite was used to fill up the micro spaces between the tooth and fracture segment. As it has the property of dentinal replacement and minimize the crack propagation, reinforcing effect of fibers with increase in upto $25 \%$ of the fiber content with excellent fracture toughness. The combined use of a glass fibre reinforced composite root canal post and an original crown fragment is a simple and efficient procedure for the treatment of traumatized anterior teeth that offers an excellent aesthetic and functional results. Due to the subgingival extension of the margin, isolation was an issue, hence full thickness mucoperiosteal flap was raised to expose the margins. Adrenaline impregnated gauze was used to maintain the haemostasis and isolation. Here, dimples were opted as a mode of an additional retention feature as simple reattachment without reinforcement obtain only $1 / 2$ of fracture strength, but with additional features it extends up to $90 \%$ of the strength.

However, many other techniques noted in literature associated limitations. These may include multi-visit appointments, cost, stabilisation (splinting), and be more invasive procedure as compared to the treatment modality chosen for this case. The single visit, multidisciplinary approach to a crown fracture tooth requires consideration of periodontal, endodontic, restorative and occlusal factors. This presents a great challenge to the dental surgeon, with regard to both clinical skills and time management. Follow up must involve assessment of occlusion, periodontium and subsequent traumatic force reduction. Every part have its own limitation which include that there is not enough scientific evidence on the long term retention of teeth. Also reattachment procedure is technique sensitive, strict isolation with adequate bonding protocols are required also in cases of complex crown/root fractures or were isolation is difficult, reattachment is questionable.

\section{Conclusion}

Reattachment of fractured tooth fragments offers a viable restorative option for the clinician because it restores tooth function and esthetics with the use of a very conservative and cost-effective approach.

\section{References}

1. Poi WR, Cardoso LC, Castro JCM, Cintra LTA, Gulinelli JL, et al. (2007) Multidisciplinary treatment approach for crown fracture and crown-root fracture-a case report. Dent Traumatol 23(1): 51-55.

2. Divakar HD, Nayak M, Shetty R (2007) Changing concepts in fracture reattachment of teeth-a case series. Endodontology 2: 27-35.

3. Taguchi CMC, Bernardon JK, Zimmermann G, Baratieri LN (2015) Tooth Fragment Reattachment: A Case Report Operative Dentistry pp: 40-42.

4. Eden E, Yanar SC, Sonmez S (2007) Reattachment of subgingivally fractured central incisor with an open apex. Dent Traumatol 23(3): 184-189.

5. Wadhwani CPK (2000) A single visit, multidisciplinary approach to the management of traumatic tooth crown fracture. Br Dent J 188(11): 593-598.

6. Olsburgh S, Jacoby T, Krejci I (2002) Crown fractures in the permanent dentition: pulpal and restorative considerations. Dent Traumatol 18(3): 103-115.

7. Murchison DF, Burke FJ, Worthington RB (1999) Incisal edge reattachment: indications for use and clinical technique. Br Dent J 186(12): 614-619.

8. Tay FR, Pashley DH (2007) Monoblocks in root canals: A hypothetical or a tangible goal. J Endod 33(4): 391-398.

9. Trope M, Maltz DO, Tronstad L (1985) Resistance to fracture of restored endodontically treated teeth. Endod Dent Traumatol 1(3): 108-111.

10. Badami AA, Dunne SM, Scheer B (1995) An in vitro investigation into the shear bond strengths of two dentine-bonding agents used in the reattachment of incisal edge fragments. Endod Dent Traumatol 11(3): 129-135. 